\title{
A NEW PROOF OF PÓLYA-KNOPP'S INEQUALITY WITH AN EXTENSION
}

\author{
ERn Gun KwON And Min Ju Jo
}

Abstract. We show that a continuous version of Hölder's inequality can give a new and direct proof of the Pólya-Knopp type inequalities. We also have a single variable generalization of Pólya-Knopp's inequality.

Mathematics subject classification (2010): Primary 26D15, 26D10.

Keywords and phrases: Hölder's inequality, Pólya-Knopp's inequality, Hardy’s inequality.

\section{REFERENCES}

[1] E. F. Beckenbach and R. Bellman, Inequalities, Berlin, Springer-Verlag, 1983.

[2] G. H. Hardy, J. E. Littlewood, G. Pólya, Inequalities 2nd ed., Cambridge University Press, 1952 (1934).

[3] K. KNopp, Über reihen mit positiven gliedern, J. London Math. Soc. 3 (1928), $205-211$.

[4] E. G. Kwon, Extension of Hölder's inequality (I), Bull. Austral. Math. Soc. 51 (1995), 369-375.

[5] E. G. KWon And E. K. BAE, On a continuous form of Hölder inequality, Jour. Math. Anal. Appl. 343 (2008), 585-592.

[6] A. ČIŽMeŠIJA, J. PEČARIĆ, L. E. PERsson, On strengthened Hardy and Pólya-Knopp's inequalities, Journal of Approximation Theory 125 (2003), 74-84.

[7] W. Rudin, Real and complex analysis 2nd ed., McGraw-Hill, New York, 1974. 